Volume 128, Number 10, Pages 3035-3041

S 0002-9939(00)05345-4

Article electronically published on April 7, 2000

\title{
MAXIMAL INEQUALITIES FOR THE ORNSTEIN-UHLENBECK PROCESS
}

\author{
S. E. GRAVERSEN AND G. PESKIR
}

(Communicated by Stanley Sawyer)

\begin{abstract}
Let $V=\left(V_{t}\right)_{t \geq 0}$ be the Ornstein-Uhlenbeck velocity process solving

$$
d V_{t}=-\beta V_{t} d t+d B_{t}
$$

with $V_{0}=0$, where $\beta>0$ and $B=\left(B_{t}\right)_{t \geq 0}$ is a standard Brownian motion. Then there exist universal constants $C_{1}>0$ and $C_{2}>0$ such that

$$
\frac{C_{1}}{\sqrt{\beta}} E \sqrt{\log (1+\beta \tau)} \leq E\left(\max _{0 \leq t \leq \tau}\left|V_{t}\right|\right) \leq \frac{C_{2}}{\sqrt{\beta}} E \sqrt{\log (1+\beta \tau)}
$$

for all stopping times $\tau$ of $V$. In particular, this yields the existence of universal constants $D_{1}>0$ and $D_{2}>0$ such that

$D_{1} E \sqrt{\log (1+\log (1+\tau))} \leq E\left(\max _{0 \leq t \leq \tau} \frac{\left|B_{t}\right|}{\sqrt{1+t}}\right) \leq D_{2} E \sqrt{\log (1+\log (1+\tau))}$

for all stopping times $\tau$ of $B$. This inequality may be viewed as a stopped law of iterated logarithm. The method of proof relies upon a variant of Lenglart's domination principle and makes use of Itô calculus.
\end{abstract}

\section{INTRODUCTION}

Consider the random movement of a Brownian particle suspended in a liquid. The Einstein-Smoluchowski theory suggests the standard Brownian motion $B_{t} \sim N(0, t)$ as a model for the position of the particle. The Ornstein-Uhlenbeck theory [6] relies upon Newtonian mechanics and suggests that the position of the Brownian particle should be modelled as $X_{t}=\int_{0}^{t} V_{r} d r$ where $V_{t}=e^{-\beta t} \int_{0}^{t} e^{\beta r} d B_{r} \sim$ $N\left(0, \frac{1}{2 \beta}\left(1-e^{-2 \beta t}\right)\right)$ is the Brownian velocity solving the Langevin equation:

$$
d V_{t}=-\beta V_{t} d t+d B_{t} \quad(\beta>0)
$$

(see [3] for more details). The Einstein-Smoluchowski theory may be seen as an idealised Ornstein-Uhlenbeck theory, and predictions of either cannot be distinguished by experiment. However, if the Brownian particle is under influence of an external force, the Einstein-Smoluchowski theory breaks down, while the OrnsteinUhlenbeck theory remains successful (see [3], pp. 53-78). Perhaps one of the main

Received by the editors May 29, 1998 and, in revised form, November 10, 1998.

2000 Mathematics Subject Classification. Primary 60J65, 60G40, 60E15; Secondary 60J60, $60 \mathrm{G} 15$.

Key words and phrases. Ornstein-Uhlenbeck velocity process, maximum process, stopping time, maximal inequality, Lenglart's domination principle, Brownian motion, diffusion process, Gaussian process, the Langevin stochastic differential equation.

The authors were supported by the Danish National Research Foundation. 
reasons that the Einstein-Smoluchowski model is so popular in stochastic calculus today is due to the fact that the standard Brownian motion is a martingale.

Consider the standard Brownian motion $B=\left(B_{t}\right)_{t \geq 0}$. Then the celebrated Burkholder-Gundy inequality [1] states that there exist universal constants $A_{1}>0$ and $A_{2}>0$ such that

$$
A_{1} E(\sqrt{\tau}) \leq E\left(\max _{0 \leq t \leq \tau}\left|B_{t}\right|\right) \leq A_{2} E(\sqrt{\tau})
$$

for all stopping times $\tau$ of $B$. In other words, and less formally, this inequality states that the maximal position of the Brownian particle, taken up to a random instant of time $\tau$ which does not anticipate the future, in average behaves as $\sqrt{\tau}$.

In this note we address the same question for the velocity process $V=\left(V_{t}\right)_{t \geq 0}$. Our main result (Theorem 2.5) shows that the maximal velocity of the Brownian particle, taken up to a random instant of time $\tau$ which does not anticipate the future, in average behaves as $\sqrt{\log (1+\tau)}$. In view of the "reverse" drift term in (1.1), which is due to a "frictional" force towards the origin (equilibrium state of velocity zero), the quantitative difference in the result is in agreement with intuition. The result of Theorem 2.5 can also be restated in terms of the standard Brownian motion $B$, and this may be viewed as a stopped law of iterated logarithm (Corollary $2.7)$.

\section{The Result And PRoOF}

The following domination principle was initially proved in 2] in the case $H(x)=$ $x^{p}$ for $0<p<1$. Its extension to more general functions $x \mapsto H(x)$ follows along the same lines and can be found in [5] (pp. 155-156). This extension appears crucial in our treatment below, and we present the proof for completeness.

Proposition 2.1 (Lenglart). Let $\left(\Omega, \mathcal{F},\left(\mathcal{F}_{t}\right)_{t \geq 0}, P\right)$ be a filtered probability space, let $X=\left(X_{t}\right)_{t \geq 0}$ be an $\left(\mathcal{F}_{t}\right)$-adapted non-negative right-continuous process, let $A=$ $\left(A_{t}\right)_{t \geq 0}$ be an $\left(\mathcal{F}_{t}\right)$-adapted increasing continuous process satisfying $A_{0}=0$, and let $H: \mathbf{R}_{+} \rightarrow \mathbf{R}_{+}$be an increasing continuous function satisfying $H(0)=0$. Assume that

$$
E\left(X_{\tau}\right) \leq E\left(A_{\tau}\right)
$$

for all bounded $\left(\mathcal{F}_{t}\right)$-stopping times $\tau$. Then

$$
E\left(\sup _{0 \leq t \leq \tau} H\left(X_{t}\right)\right) \leq E\left(\widetilde{H}\left(A_{\tau}\right)\right)
$$

for all $\left(\mathcal{F}_{t}\right)$-stopping times $\tau$, where

$$
\widetilde{H}(x)=x \int_{x}^{\infty} \frac{1}{s} d H(s)+2 H(x)
$$

for all $x \geq 0$.

Proof. By Fubini's theorem we obtain

$$
\begin{aligned}
E\left(\sup _{0 \leq t \leq \tau} H\left(X_{t}\right)\right) & \leq E\left(H\left(\sup _{0 \leq t \leq \tau} X_{t}\right)\right)=E\left(\int_{0}^{\infty} 1_{\left\{\sup _{0 \leq t \leq \tau} X_{t}>s\right\}} d H(s)\right) \\
& \leq \int_{0}^{\infty}\left(P\left\{\sup _{0 \leq t \leq \tau} X_{t}>s, A_{\tau} \leq s\right\}+P\left\{A_{\tau}>s\right\}\right) d H(s)
\end{aligned}
$$


since $s \mapsto H(s)$ is increasing and continuous. Consider the following stopping times:

$$
\begin{aligned}
& \tau_{1}=\inf \left\{t>0 \mid X_{t}>s\right\}, \\
& \tau_{2}=\inf \left\{t>0 \mid A_{t}>s\right\} .
\end{aligned}
$$

Then by Markov's inequality and (2.1) we find:

$$
\begin{aligned}
P\left\{\sup _{0 \leq t \leq \tau} X_{t}>s, A_{\tau} \leq s\right\} & \leq P\left\{\tau_{1} \leq \tau, \tau_{2} \geq \tau\right\} \leq P\left\{X_{\tau_{1} \wedge \tau_{2} \wedge \tau} \geq s\right\} \\
& \leq \frac{1}{s} E\left(A_{\tau_{1} \wedge \tau_{2} \wedge \tau}\right)
\end{aligned}
$$

whenever $\tau$ is bounded. From (2.4) and (2.6) we can conclude:

$$
\begin{aligned}
E\left(\sup _{0 \leq t \leq \tau} H\left(X_{t}\right)\right) & \leq \int_{0}^{\infty}\left(\frac{1}{s} E\left(A_{\tau} 1_{\left\{A_{\tau} \leq s\right\}}\right)+2 P\left\{A_{\tau}>s\right\}\right) d H(s) \\
& \leq E\left(A_{\tau} \int_{A_{\tau}}^{\infty} \frac{1}{s} d H(s)\right)+2 E\left(H\left(A_{\tau}\right)\right)=E\left(\widetilde{H}\left(A_{\tau}\right)\right)
\end{aligned}
$$

for all bounded $\tau$. Finally, observe that $x \mapsto \widetilde{H}(x)$ is increasing, and pass to the limit when $k \rightarrow \infty$ to reach any $\tau$ through bounded ones $\tau \wedge k$. This completes the proof.

Remark 2.2. If $H(x)=x^{p}$ with $0<p<1$, then $\widetilde{H}(x)=((2-p) /(1-p)) x^{p}$; if $H(x)=x$, then $\widetilde{H}(x) \equiv+\infty$, and the bound on the right-hand side in $(2.2)$ is non-interesting. Generally, the right-hand side in (2.2) gives a non-trivial bound if $H(x)$ tends to infinity as slow as $x^{p}$ for some $0<p<1$; the bound is better (asymptotically optimal) if the error in (2.1) is smaller (negligible).

1. The initial result which we state now is motivated by the considerations in 4 . This is addressed in more detail in Remark 2.4 following the proof below.

Theorem 2.3. Let $V=\left(V_{t}\right)_{t \geq 0}$ be the Ornstein-Uhlenbeck velocity process solving (1.1) with $V_{0}=0$, where $B=\left(B_{t}\right)_{t \geq 0}$ is a standard Brownian motion. Introduce the following functional:

$$
I_{t}=\int_{0}^{t} e^{\beta V_{r}^{2}} d r
$$

Then there exist universal constants $A_{1}>0$ and $A_{2}>0$ such that

$$
\frac{A_{1}}{\sqrt{\beta}} E \sqrt{\log \left(1+\beta I_{\tau}\right)} \leq E\left(\max _{0 \leq t \leq \tau}\left|V_{t}\right|\right) \leq \frac{A_{2}}{\sqrt{\beta}} E \sqrt{\log \left(1+\beta I_{\tau}\right)}
$$

for all stopping times $\tau$ of $V$.

Proof. If $x \mapsto F(x)$ is even and $C^{2}$, then by Itô's formula we find:

$$
F\left(\left|V_{t}\right|\right)=F(0)+\int_{0}^{t}\left(\mathbb{L}_{V} F\right)\left(V_{r}\right) d r+\int_{0}^{t} F^{\prime}\left(V_{r}\right) d B_{r}
$$

where $\mathbb{L}_{V}$ denotes the infinitesimal generator of $V$ :

$$
\mathbb{L}_{V}=-\beta v \frac{\partial}{\partial v}+\frac{1}{2} \frac{\partial^{2}}{\partial v^{2}} .
$$


Motivated by our considerations in 4, we shall set

$$
F(v)=\frac{1}{\beta}\left(e^{\beta v^{2}}-1\right) .
$$

Then it is easily verified that $\mathbb{L}_{V}(F)=c$ where $c(v)=e^{\beta v^{2}}$. By applying the optional sampling theorem in (2.10), it follows that

$$
E\left(F\left(\left|V_{\tau}\right|\right)\right)=E\left(I_{\tau}\right)
$$

for all bounded stopping times $\tau$ of $V$. This shows that the condition (2.1) is satisfied with $X_{t}=F\left(\left|V_{t}\right|\right)$ and $A_{t}=I_{t}$. Denote $H(x)=F^{-1}(x)$ and observe that

$$
H(x)=H(x ; \beta)=\frac{1}{\sqrt{\beta}} \sqrt{\log (1+\beta x)}
$$

where by $H(x ; \beta)$ we indicate the dependence on $\beta$. By (2.3) we then have

$$
\widetilde{H}(x ; \beta)=x \int_{x}^{\infty} \frac{1}{s} d H(s ; \beta)+2 H(x ; \beta) .
$$

Consider the following function:

$$
G(x ; \beta)=\frac{x}{H(x ; \beta)} \int_{x}^{\infty} \frac{1}{s} d H(s ; \beta) .
$$

Observe that for all $x$ we have:

$$
G(x ; \beta)=G(\beta x ; 1) .
$$

Thus, if we want to compute the limit of $G(x ; \beta)$ when $x \rightarrow \infty$ or $x \rightarrow 0$, it is no restriction to assume that $\beta=1$. Note that

$$
G(x ; 1)=\frac{x}{2 \sqrt{\log (1+x)}} \int_{x}^{\infty} \frac{d s}{\sqrt{\log (1+s)}(1+s) s} .
$$

Elementary calculations show that

$$
\begin{aligned}
& \lim _{x \rightarrow 0} G(x ; 1)=1, \\
& \lim _{x \rightarrow \infty} G(x ; 1)=0, \\
& 0 \leq G(x ; 1) \leq 1 \quad(\forall x>0) .
\end{aligned}
$$

From (2.15) we then find:

$$
\widetilde{H}(x ; \beta) \leq 3 H(x ; \beta)
$$

for all $x \geq 0$, and hence the right-hand inequality in (2.9) follows from (2.2) and (2.14).

To prove the left-hand inequality in (2.9), we shall note by (2.13) that

$$
E\left(I_{\tau}\right) \leq E\left(\max _{0 \leq t \leq \tau} F\left(\left|V_{t}\right|\right)\right)
$$

for all bounded stopping times $\tau$ of $V$. Thus, the left-hand inequality in (2.9) follows from (2.2) and (2.14) upon the identification $X_{t}=I_{t}$ and $A_{t}=\max _{0 \leq r \leq t} F\left(\left|V_{r}\right|\right)$. The proof is complete. 
Remark 2.4. It was proved in [4] that

$$
E\left(\max _{0 \leq t \leq \tau}\left|V_{t}\right|\right) \leq \frac{C}{\sqrt{\beta}} \sqrt{\log E\left(e^{\beta V_{\tau}^{2}}\right)}
$$

for all stopping times $\tau$ of $V$ for which the process $\left(e^{\beta V_{\tau \wedge t}^{2}}\right)_{t \geq 0}$ is uniformly integrable; by Itô's formula this inequality is equivalently written as follows:

$$
E\left(\max _{0 \leq t \leq \tau}\left|V_{t}\right|\right) \leq \frac{C}{\sqrt{\beta}} \sqrt{\log \left(1+\beta E\left(I_{\tau}\right)\right)}
$$

where $C>0$ is some constant. Our result (2.9) shows that the second expectation sign in (2.25) can be pulled out in front of the square-root and logarithm sign; in view of Jensen's inequality this bound is better, although not easily computed; as the inequality (2.9) above is two-sided, this also detects the real size of the error in the terminal-value bound (2.24); observe also that our proof above establishes (2.9) with $A_{1}=1 / 3$ and $A_{2}=3$; thus $C$ in $(2.24)$ can be taken as 3 .

2. A main disadvantage of the inequality (2.9) is the complicated form of the functional $I_{\tau}$. In our attempt to understand better its size, we now prove that $I_{\tau}$ in (2.9) can be replaced by $\tau$. In view of the obvious inequality $I_{\tau} \geq \tau$, and that $I_{\tau}$ is actually much larger than $\tau$, this fact may seem surprising at first. However, noting that we also have the logarithm function in (2.9), and recalling that the variance of $V_{t} \sim N\left(0, \frac{1}{2 \beta}\left(1-e^{-2 \beta t}\right)\right)$ remains bounded over all $t$, we see that everything agrees well with intuition.

Theorem 2.5. Let $V=\left(V_{t}\right)_{t \geq 0}$ be the Ornstein-Uhlenbeck velocity process solving (1.1) with $V_{0}=0$, where $B=\left(B_{t}\right)_{t \geq 0}$ is a standard Brownian motion. Then there exist universal constants $C_{1}>0$ and $C_{2}>0$ such that

$$
\frac{C_{1}}{\sqrt{\beta}} E \sqrt{\log (1+\beta \tau)} \leq E\left(\max _{0 \leq t \leq \tau}\left|V_{t}\right|\right) \leq \frac{C_{2}}{\sqrt{\beta}} E \sqrt{\log (1+\beta \tau)}
$$

for all stopping times $\tau$ of $V$.

Proof. If $x \mapsto F(x)$ is even and $C^{2}$, then by Itô's formula we know that (2.10) holds. Motivated by this expression, consider the equation:

$$
\mathbb{L}_{V}(F)=1
$$

with $\mathbb{L}_{V}$ as in (2.11). The general solution of (2.27) is given by

$$
F(x)=\int_{0}^{x} e^{\beta u^{2}}\left(2 \int_{0}^{u} e^{-\beta v^{2}} d v+K_{1}\right) d u+K_{2}
$$

where $K_{1}$ and $K_{2}$ are constants. Motivated by the fact that $A$ from Proposition 2.1 should satisfy $A_{0}=0$, we shall impose the condition $F(0)=0$, which implies that $K_{2}=0$. Imposing further that $F^{\prime}(0)=0$, which implies that $K_{1}=0$, we obtain the following solution of (2.27):

$$
F(x)=2 \int_{0}^{x} e^{\beta u^{2}} \int_{0}^{u} e^{-\beta v^{2}} d v d u
$$

Observe that $x \mapsto F(x)$ is even and $C^{2}$, and thus (2.10) holds.

Applying the optional sampling theorem in (2.10), and using (2.27), we see that

$$
E\left(F\left(\left|V_{\tau}\right|\right)\right)=E(\tau)
$$


for all bounded stopping times $\tau$ of $V$. Thus the condition (2.1) is satisfied with $X_{t}=F\left(\left|V_{t}\right|\right)$ and $A_{t}=t$. From (2.30) we also see that

$$
E(\tau) \leq E\left(\max _{0 \leq t \leq \tau} F\left(\left|V_{t}\right|\right)\right)
$$

for all bounded stopping times $\tau$ of $V$. Thus the condition (2.1) is also satisfied with $X_{t}=t$ and $A_{t}=\max _{0<r<t} F\left(\left|V_{r}\right|\right)$.

Denoting $H(x)=F^{-1}(x)$, it is possible to prove that

$$
\frac{1}{\sqrt{\beta}} \sqrt{\log (1+\beta x)} \leq H(x) \leq \frac{D}{\sqrt{\beta}} \sqrt{\log (1+\beta x)}
$$

for all $x \geq 0$, where $D>1$ is some constant. The left-hand side in (2.32) is verified straightforwardly, while the right-hand side requires some more effort. Our calculations show that one may take $D=1.1265 \ldots$.

The result now follows from (2.30)-(2.32) and (2.2) above upon verifying that $\widetilde{H}(x) / H(x) \leq 3$ for all $x>0$; observe that $F(x) \geq x^{2}$ and $F^{\prime}(x) \geq 2 x$ so that $H(x) \leq \sqrt{x}$ and $H^{\prime}(x) \leq 1 /(2 \sqrt{x})$ for all $x>0$. The proof is complete.

Remark 2.6. Observe from the proof above that in (2.26) one may take $C_{1}=1 / 3$ and $C_{2}=3 D=3.3795 \ldots$. Note also that $(1.2)$ is obtained from (2.26) by letting $\beta \downarrow 0$.

Corollary 2.7. Let $B=\left(B_{t}\right)_{t \geq 0}$ be standard Brownian motion. Then there exist universal constants $D_{1}>0$ and $D_{2}>0$ such that

$$
D_{1} E \sqrt{\log (1+\log (1+\tau))} \leq E\left(\max _{0 \leq t \leq \tau} \frac{\left|B_{t}\right|}{\sqrt{1+t}}\right) \leq D_{2} E \sqrt{\log (1+\log (1+\tau))}
$$

for all stopping times $\tau$ of $B$.

Proof. In the setting of Theorem 2.5 above, we shall use the well-known fact that

$$
V_{t}=\frac{1}{\sqrt{2 \beta}} e^{-\beta t} B\left(e^{2 \beta t}-1\right)
$$

is an Ornstein-Uhlenbeck process. Set $\sigma_{t}=e^{2 \beta t}-1$; then $\tau$ is a stopping time of $V$ if and only if $\sigma_{\tau}$ is a stopping time of $B$. From (2.34) we see that

$$
\sqrt{2 \beta}\left|V_{t}\right|=\frac{\left|B_{\sigma_{t}}\right|}{\sqrt{1+\sigma_{t}}}
$$

Set $H(x ; \beta)=(1 / \sqrt{\beta}) \sqrt{\log (1+\beta x)}$; then $(2.26)$ above can equivalently be rewritten as follows:

$$
E\left(\max _{0 \leq t \leq \tau} \frac{\left|B_{\sigma_{t}}\right|}{\sqrt{1+\sigma_{t}}}\right) \sim \sqrt{2 \beta} E(H(\tau ; \beta)) .
$$

Substituting $\sigma_{t}=u$ in (2.36), we see that

$$
E\left(\max _{0 \leq u \leq \sigma(\tau)} \frac{\left|B_{u}\right|}{\sqrt{1+u}}\right) \sim \sqrt{2 \beta} E\left(H\left(\sigma^{-1}(\sigma(\tau)) ; \beta\right)\right) .
$$

Observe that $\sqrt{2 \beta} H(x ; \beta)=\sqrt{2} \sqrt{\log (1+\beta x)}$ and $\sigma^{-1}(u)=(1 / 2 \beta) \log (1+u)$. Thus

$$
\sqrt{2 \beta} H\left(\sigma^{-1}(\widetilde{\tau}) ; \beta\right)=\sqrt{2} \sqrt{\log \left(1+\frac{1}{2} \log (1+\widetilde{\tau})\right)}
$$


where $\widetilde{\tau}=\sigma(\tau)$ is a stopping time of $B$. However, since clearly

$$
\log \left(1+\frac{1}{2} \log (1+x)\right) \sim \log (1+\log (1+x))
$$

when $x$ tends to 0 or $\infty$, we see from (2.37) and (2.38) that (2.33) holds. The proof is complete.

Corollary 2.8. Let $M=\left(M_{t}\right)_{t \geq 0}$ be a continuous local martingale with the quadratic variation process $\left(\langle M\rangle_{t}\right)_{t \geq 0}$. Then there exist universal constants $D_{1}>0$ and $D_{2}>0$ such that

$$
\begin{aligned}
D_{1} E \sqrt{\log \left(1+\log \left(1+\langle M\rangle_{\tau}\right)\right)} & \leq E\left(\max _{0 \leq t \leq \tau} \frac{\left|M_{t}\right|}{\sqrt{1+\langle M\rangle_{t}}}\right) \\
& \leq D_{2} E \sqrt{\log \left(1+\log \left(1+\langle M\rangle_{\tau}\right)\right)}
\end{aligned}
$$

for all stopping times $\tau$ of $M$.

Proof. It follows from Corollary 2.7 by a standard time-change argument (see [5]).

\section{REFERENCES}

[1] Burkholder, D. L. and Gundy, R. F. (1970). Extrapolation and interpolation of quasi-linear operators on martingales. Acta Math. 124 (249-304). MR 55:13567

[2] Lenglart, E. (1977). Relation de domination entre deux processus. Ann. Inst. H. Poincaré Probab. Statist. 13 (171-179). MR 57:10810

[3] Nelson, E. (1967). Dynamical Theories of Brownian Motion. Princeton Univ. Press. MR 35:5001

[4] Peskir, G. (1998). Controlling the velocity of Brownian motion by its terminal value. Research Report No. 391, Dept. Theoret. Statist. Aarhus (11 pp). Analytic and Geometric Inequalities and their Applications (eds. T. M. Rassias and H. M. Srivastava), Math. Appl., Vol. 478, Kluwer Acad. Publ., Dordrecht, 1999 (323-333).

[5] Revuz, D. and Yor, M. (1994). Continuous Martingales and Brownian Motion. SpringerVerlag. MR 95h:60072

[6] Uhlenbeck, G. E. and Ornstein, L. S. (1930). On the theory of Brownian motion. Physical Review 36 (823-841).

Institute of Mathematics, University of Aarhus, Ny Munkegade, 8000 Aarhus, DenMARK

E-mail address: matseg@imf.au.dk

Institute of Mathematics, University of Aarhus, Ny Munkegade, 8000 Aarhus, Denmark (Department of Mathematics, University of Zagreb, BijeničKa 30, 10000 Zagreb, CROATIA)

E-mail address: goran@imf.au.dk

$U R L:$ http://www.imf.au.dk/ goran 